\title{
Avaliação de um Programa de Intervenção de Habilidades Sociais Educativas Parentais: Um Estudo-Piloto
}

Evaluation of an intervention of parental educative social skills program : A pilot study

Alessandra Turini Bolsoni-Silva Universidade Estadual Paulista

Alessandra Salina-Brandão Universidade Federal Paulista

Fabiana Maris Versuti-Stoque Universidade Estadual Paulista

Andréa Regina Rosin-Pinola Universidade de São Paulo

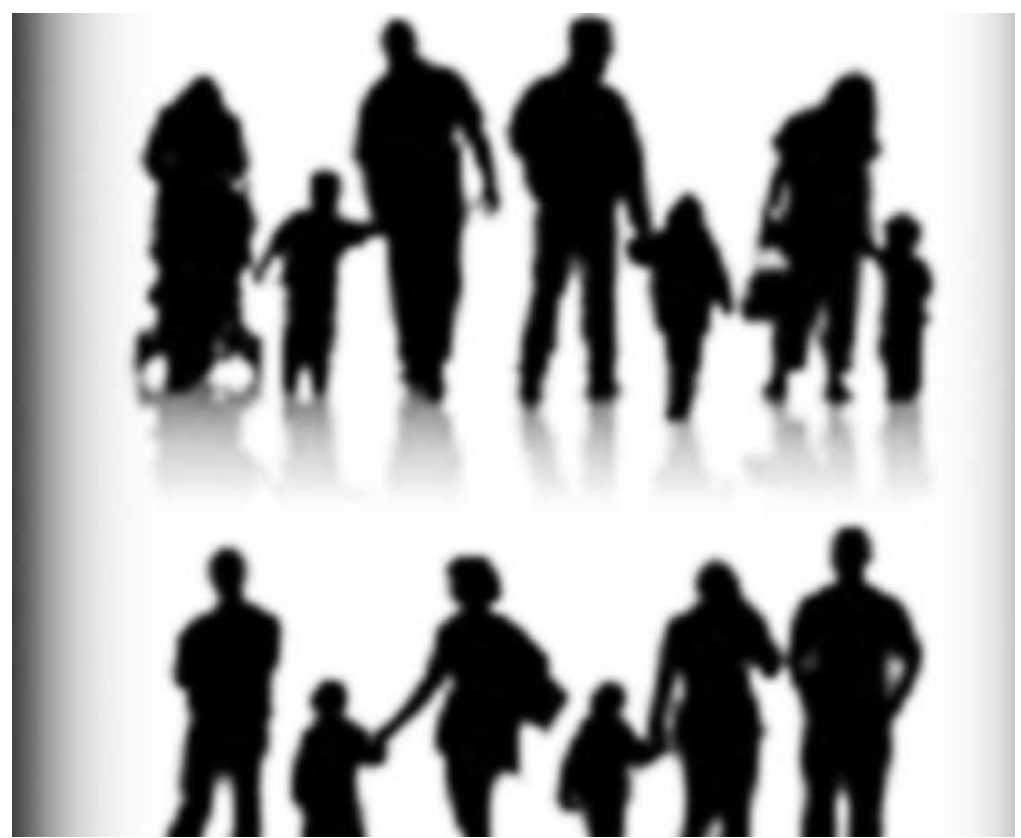


Resumo: Estudos têm apontado relações entre déficits de habilidades sociais educativas de pais e problemas de comportamento de seus filhos. Nesse sentido, esta pesquisa visa a descrever os efeitos de um procedimento de intervenção com pais, o qual pretendeu promover suas habilidades sociais educativas. Participaram dois pais e sete mães, que elegeram o filho com o qual descreveram ter maiores dificuldades de relacionamento. Para a avaliação: 1) das dificuldades dos pais, foi utilizada uma entrevista estruturada, e 2) dos procedimentos de intervenção, foram utilizados: a) questionário de habilidades sociais educativas parentais (QHSE-P) e b) inventário de habilidades sociais (IHS-Del Prette). Os resultados das comparações pré e pós-intervenção do IHS-Del Prette mostraram aumento no escore dos grupos; as comparações do QHSE-P apontaram aquisições de diversas habilidades sociais educativas: expressar sentimentos positivos, agradecer elogios, dizer não e negociar limites. Discute-se a necessidade de procedimentos de promoção de habilidades sociais educativas para ampliar o repertório social parental.

Palavras-chave: Treinamento de habilidades sociais. Habilidades sociais educativas. Intervenção com pais. Interação pais-filhos.

Abstract: Studies have pointed the relations between the deficit of social education skills of the parents and the behavior problem of their children. In relation to this subject, this research has the objective of describing the effects of the intervention process in parents aiming to promote their social educative skills. Two fathers and seven mothers contributed with this research choosing among their children the one with whom they have more relationship difficulties. For the evaluation: 1) for the parents' difficulties, a structured interview was used and 2) for the intervention procedures, it was used a) a parental social educative skills questionnaire and b) a list of social skills. The results of the comparisons made before and after the intervention of the IHS-Del Prette show a rising on the score of the groups; in the comparisons of the QHSE-P it was verified the acquisitions of educative social skills: to express positive feelings, to thank praises, to deny and negotiate limits. It is discussed the need of procedures that may lead to the promotion of educative social skills as a way to increase the social knowledge of the parents.

Keywords: Training of social abilities. Educative social skills. Intervention with parents. Interaction parents-children.

Pesquisas nacionais e internacionais (por ex. Brestan, Jacobs, Rayfield \& Eyberg, 1999; Marinho, 1999; McMahon, 1996; Roca \& Brandão, 1997; Ruma, Burke, Thompson, 1996; Silva, Del Prette \& Del Prette, 2000) descrevem intervenções que têm sido realizadas com o objetivo de ajudar pais e mães a melhorar o relacionamento com seus filhos. Comumente, os pais que participam desses trabalhos relatam que os filhos apresentam problemas de comportamento, especialmente agressividade e desobediência (Kaplan, Sadock, \& Grebb, 1997).

Patterson, Reid e Dishion (2002) discutem sobre a relação de dependência entre práticas educativas parentais e problemas de comportamento de filhos. Os autores afirmam que práticas parentais positivas podem evitar o surgimento e/ou a manutenção de dificuldades de interação, e, por outro lado, as negativas podem aumentar a probabilidade de sua ocorrência. Gomide (2006) descreve que as práticas educativas positivas compreendem a monitoria positiva (que envolve, por exemplo, o uso adequado da atenção e a distribuição de privilégios) e o comportamento moral (que implica, por exemplo, promover condições favoráveis ao desenvolvimento das virtudes, tais como empatia, senso de justiça, responsabilidade e trabalho). As práticas educativas negativas 
envolvem a negligência, a ausência de atenção e de afeto e a disciplina relaxada, que compreende, entre outros aspectos, o relaxamento das regras estabelecidas e a punição inconsistente.

Essas definições remetem ao campo teóricoprático do treinamento em habilidades sociais (THS) no que diz respeito a identificar as habilidades sociais envolvidas nas práticas educativas, sejam positivas ou negativas. Del Prette e Del Prette (2001) salientam que as habilidades sociais educativas (HSE) são aquelas intencionalmente voltadas para a promoção do desenvolvimento e da aprendizagem do outro, em situação formal ou informal. Silva (2000) define o termo habilidades sociais educativas parentais (HSE-P) como o conjunto de habilidades sociais dos pais aplicáveis à prática educativa dos filhos e exemplifica que a HSE-P de estabelecer limites e/ou regras pode envolver outras habilidades, tais como dizer não e solicitar mudança de comportamento.

Os artigos da literatura internacional sobre treinamento de pais podem ser categorizados em dois grupos: trabalhos que apresentam mais um caráter remediativo que preventivo e trabalhos que ressaltam o treino de habilidades parentais como um fator de prevenção para os problemas de comportamento de filhos. O primeiro conjunto de artigos enfatiza o manejo de comportamentos-problema das crianças (Brestan et al., 1999; Dishion \& Andrews, 1995; Jouriles et al., 2001; Ruma, Burke, \& Thompson, 1996; Sanders, MarkieDadds, Tully, \& Bor, 2000), através de técnicas utilizadas para modificar comportamentos, tais como time-out, ignorar comportamento inadequado e elogiar comportamento adequado considerado incompatível. Em relação ao segundo conjunto de trabalhos, Dishion e Andrews (1995) realizaram um estudo com o objetivo de ensinar habilidades de comunicação; Jouriles et al. (2001) e Sanders et al. (2000) realizaram intervenções para promover relacionamento positivo entre pais e filhos. De modo geral, as intervenções ocorrem em grupo (Brestan et al., 1999; Cobham, Dadds, \& Spence, 1998; Dishion \& Andrews, 1995; Peterson, Tremblay \& Ewigman, 2003; Ruma et al., 1996; Sanders et al., 2000; Taylor, Schmidt, Pepler e Hodgins, 1998) e uma vez por semana, variando de 10 a 16 sessões (Brestan et al. 1999; Cobham et al., 1998; Dadds \& Spence, 1998; Dishion \& Andrews, 1995; Peterson et al., 2003; Pfiffner \& McBurnett, 1997; Ruma et al., 1996; Sanders et al., 2000; Taylor et al., 1998).

Nota-se uma ampla literatura internacional relacionada à intervenção com pais de crianças com problemas de comportamento. Nacionalmente, há também pesquisadores preocupados com essa temática (por ex., Marinho, 1999; Silva et al., 2000).

Marinho (1999) também promoveu um treinamento com 12 pais de crianças com idades entre 7 e 12 anos com problemas de comportamento. A intervenção, baseada nos trabalhos de Patterson (1974, citado por Marinho, 1999) e de Forehand e McMahon (1981, citado por Marinho, 1999), era composta por 12 sessões, de aproximadamente 90 minutos, e foi organizada em três fases: a) atenção diferencial (cinco sessões), em que os pais foram ensinados a atentar e a reagir de outro modo em relação aos comportamentos habilidosos e indicativos de problemas; b) treino em solução de problemas (cinco sessões), para que os pais pudessem realizar adequadamente análises de contingências e elaborar planos de ações e c) assuntos diversos (duas sessões) para discutir temas de interesse dos pais, tais como sexualidade, drogas e religião. Marinho (1999) afirma que o programa foi efetivo em desenvolver habilidades que ajudaram os pais a reduzir comportamentos delinqüentes e agressivos.

Rocha e Brandão (1997) propuseram um programa para pais com quatro etapas. 
A hipótese

subjacente a este trabalho é a de que, ao aumentar a freqüência de habilidades sociais educativas parentais, estas possam prevenir a ocorrência de problemas de comportamento dos filhos, uma vez que os participantes do treinamento poderão

utilizar práticas educativas mais eficazes junto a suas crianças e/ ou adolescentes, por exemplo, utilizando reforçamento diferencial para estabelecer limites em detrimento de práticas punitivas bem como oferecendo modelos de comportamentos socialmente habilidosos.
Na primeira, os pais falavam sobre os comportamentos dos filhos. Num segundo momento, o manejo terapêutico implicava ajudar os pais a reconhecer a influência que exerciam no comportamento dos filhos. $\mathrm{Na}$ terceira etapa do trabalho, era solicitado que falassem sobre si mesmos. Finalmente, os pais eram orientados a estabelecer novas contingências, diferenciando seus comportamentos que promoviam repertório socialmente habilidoso e os que promoviam comportamentos-problema nas crianças.

Silva et al. (2000) descreveram os efeitos de um programa para pais cujos filhos não tinham problemas de comportamento. $\mathrm{O}$ treinamento em habilidades sociais foi realizado em 10 sessões estruturadas, duas vezes por semana, com duração aproximada de uma hora e meia cada. Foram treinadas habilidades sociais educativas relacionadas à comunicação, às assertivas de enfrentamento, à empatia, ao manejo comportamental e à expressão de sentimento positivo. $\mathrm{O}$ programa mostrou-se efetivo em desenvolver habilidades essenciais para promover um relacionamento positivo entre pais e filhos, isto é, habilidades de aproximação e expressão positiva, com destaque para os elogios e o agradecimento a elogios, conversar, pedir ajuda, favores e desculparse, além de estabelecer limites e dar ordens. No entanto, os pais relataram a persistência de dificuldades em deixar o uso de punições e em expressar adequadamente sentimentos negativos, no que tange a estabelecer limites, mas relataram ter consciência de tais dificuldades e de estarem esforçando-se para superá-las.

Os estudos realizados nacionalmente, ainda que escassos, possuem tendência semelhante aos internacionais ora mencionados; também se preocupam, por um lado, em ensinar os pais a manejar comportamentos-problema das crianças (Marinho, 1999; Silva et al., 2000) e, por outro, em desenvolver habilidades de auto-observação (Rocha \& Brandão, 1997), resolução de problemas (Marinho, 1999) e habilidades sociais (Silva et al., 2000).

Considerando que as interações estabelecidas entre pais e filhos são evidentemente de natureza social, o campo teórico-prático do treinamento de habilidades sociais (THS) tem sido utilizado, ainda que de maneira reduzida, para avaliar repertório social de pais e filhos e para promovê-los (Bolsoni-Silva, 2003; Bolsoni-Silva, Del Prette \& Oishi, 2003; Bolsoni-Silva \& Marturano, 2002; BolsoniSilva \& Del Prette, 2002; Silva, 2000).

A hipótese subjacente a este trabalho é a de que, ao aumentar a freqüência de habilidades sociais educativas parentais, estas possam prevenir a ocorrência de problemas de comportamento dos filhos, uma vez que os participantes do treinamento poderão utilizar práticas educativas mais eficazes junto a suas crianças e/ou adolescentes, por exemplo, utilizando reforçamento diferencial para estabelecer limites em detrimento de práticas punitivas bem como oferecendo modelos de comportamentos socialmente habilidosos.

Bolsoni-Silva (2003) concluiu que habilidades utilizadas para estabelecer limites (p. ex. dizer não, estabelecer regras, elogiar comportamentos habilidosos e solicitar mudança de comportamento) eram importantes para a avaliação do surgimento e/ou da manutenção de problemas de comportamento; por outro lado, parecem ainda mais cruciais outras habilidades, tais 
como a expressão de sentimentos e de opiniões, fazer perguntas, ter consistência nas práticas educativas parentais e concordância parental quanto à forma de educar, que foram mais relatadas por pais e por mães de crianças sem dificuldades interpessoais quando comparadas a crianças com indicativos de problemas de comportamento.

A pesquisa de Silva et al. (2000), ainda que tenha promovido habilidades sociais educativas mediante 10 sessões, não foi suficiente para alterar habilidades de estabelecer limites sem utilizar práticas coercitivas (bater, gritar, castigar, retirar privilégios) (Sidman, 1995). Acredita-se que o número reduzido de sessões e a estruturação da intervenção, com pequena oportunidade para discutir os acontecimentos da semana e realizar análises funcionais, possam ter contribuído para a permanência de algumas dificuldades, o que justifica novas tentativas nessa direção de forma a ensinar habilidades sociais educativas, seja para a melhoria da relação estabelecida entre pais e filhos, seja para o estabelecimento de limites, valendose de habilidades sociais de comunicação e expressividade, como sugerem Bolsoni-Silva e Marturano (2006).

Para tentar suprir as limitações do estudo de Silva et al. (2000), esta pesquisa visa a descrever os efeitos de uma intervenção com pais e mães para promover habilidades sociais educativas mediante comparações pré e pós-teste.

\section{Método}

\section{Participantes}

Participaram do estudo sete mães e dois pais que buscaram atendimento em um Centro de Psicologia Aplicada (CPA) de uma cidade do interior de São Paulo, com a intenção de melhorar as interações com seus filhos. Essas pessoas são consideradas não clínicas, uma vez que não haviam previamente procurado atendimento para si mesmas ou para seus filhos junto ao Centro de Psicologia Aplicada; inscreveram-se a partir da divulgação deste trabalho.

A amostra foi constituída por dois grupos de pais, conforme mostra a Tabela 1 , sendo o primeiro composto apenas por mães (denominadas P1, P2, P3, P4, P5) e o segundo, por duas mães (P6 e P9) e dois pais (P7 e P8). A idade dos participantes variou de 35 a 50 anos ( $M=43$ anos). Com exceção de uma participante (P4), todos os demais relataram possuir uma profissão. $\mathrm{O}$ número de filhos variou de dois a quatro anos, e a idade dos participantes variou de 2 a $28 \operatorname{anos}(M=12,5)$.

Outras características foram consideradas: grau de instrução, situação conjugal e renda familiar. A maioria da amostra possuía 3o grau completo (P1, P2, P5, P6, P9); P8 tinha $3^{\circ}$ grau incompleto; P3 e P7, 2o grau incompleto, e P4 tinha 10 grau incompleto. Sete participantes eram casados, P4 possuía relacionamento conjugal sem legalização e P9 era solteira. P6 e P7 eram casados. Com exceção de P4 e de P9, todos os participantes estavam empregados no período de intervenção. A renda dos participantes era a seguinte: menos de R\$ 500,00 (P9); R\$ 500,00 e $1.000,00$ (P4); $R \$ 1.100,00$ e $1.500,00$ $(\mathrm{P} 5) ; \mathrm{R} \$ 1.600,00$ e $2.000,00$ (P3); R\$ $2.100,00$ e $2.500,00$ (P6, P7); R\$2.600,00 a $3.000,00(P 8) ; R \$ 3.600,00$ e $4.000,00$ (P1, P2). 
A Tabela 1 apresenta a caracterização dos participantes e de seus filhos.

Tabela 1: Caracterização dos participantes quanto à idade, ocupação, número de filhos, idade e sexo dos mesmos.

\begin{tabular}{|c|c|c|c|c|}
\hline & PARTICIPANTES & IDADE & OCUPAÇÃO & FILHOS: SEXO/ IDADE \\
\hline & P1 - Mãe & 40 & Enfermeira & $\mathrm{F}(8,5 \text { anos })^{*}$ \\
\hline \multirow[t]{4}{*}{ GRUPO 1} & P2 - Mãe & 35 & Profâa universitária & $\mathrm{F}(2,3 \text { anos })^{*}$ \\
\hline & P3 - Mãe & 38 & Comerciante & $M(23), M(18) M(17)^{*}$ \\
\hline & P4 - Mãe & 46 & Do lar & $F(25), M(22)$ e $M(14)^{*}$ \\
\hline & P5 - Mãe & 49 & Culinarista & $\mathrm{F}(22)^{*}$ e $\mathrm{M}(17)$ \\
\hline \multirow[t]{4}{*}{ GRUPO 2} & P6 - Mãe & 42 & Assistente administrativa & $M(2) F(7)^{*}$ \\
\hline & P7 - Pai & 50 & Eletricista & $M(20), F(15)^{*}, F(7), M(2)$ \\
\hline & P8 - Pai & 40 & Representante de vendas & $M(5) *$ E M (8) \\
\hline & P9 - Mãe & 48 & Assistente social & $M(13) *$ E F (28) \\
\hline
\end{tabular}

(*) Filhos(as) que motivaram a busca pelo atendimento.

\section{Instrumentos e materiais}

Para as medidas pré e pós-intervenção, foram utilizados: a) questionário de habilidades sociais educativas parentais (QHSE-P, adaptação de Bolsoni-Silva, 2003), que tem o objetivo de avaliar a freqüência de habilidades sociais educativas, bem como de algumas especificidades (p.ex. situações/assuntos), para o(a) filho(a) que mais motivou o atendimento; b) inventário de habilidades sociais (IHS-Del Prette, Del Prette \& Del Prette, 2001a).

Uma entrevista estruturada foi realizada, na primeira sessão de avaliação, com o objetivo de investigar queixas, dificuldades e variáveis antecedentes e conseqüentes relacionadas. Essa entrevista também buscou verificar questões acerca de história prévia de atendimento psicológico e/ou psiquiátrico dos pais e de seus filhos, de forma a orientar a elaboração de estudos de caso que contivessem objetivos específicos para cada participante dos grupos.

Foram utilizados gravador e fitas-cassete para a coleta de dados (pré e pós-teste), a fim de possibilitar a transcrição literal das respostas dos participantes à entrevista inicial e ao QHSE-P; as gravações em áudio foram mantidas durante as sessões de intervenção, maximizando dados para a supervisão dos atendimentos.

\section{Procedimento}

Para a formação dos grupos, foram utilizadas diferentes estratégias de divulgação: a) cartazes (colocados no campus da Universidade e em outros locais na cidade); b) Rádio Universitária; c) site da Universidade; d) palestras ministradas pela primeira autora. Todas as estratégias de divulgação anunciavam que se tratava de uma intervenção para melhorar as relações estabelecidas entre pais e filhos e para discutir alternativas de resolução de problemas e de estabelecimento de limites. As pessoas interessadas inscreviam-se no CPA (Centro de Psicologia Aplicada de uma clínica-escola) para, em seguida, serem encaminhadas ao grupo de supervisão, sob a orientação da primeira autora. Dessa forma, os grupos foram organizados para atender a maioria das 
Destaca-se a

relevância das avaliações

diagnósticas,

que permitiram conhecer

previamente $\mathrm{O}$ repertório de

habilidades sociais educativas dos participantes, colaborando na elaboração do programa no que diz respeito ao ajuste das necessidades de cada membro do grupo. pessoas interessadas, uma vez que algumas não puderam freqüentar o atendimento nos horários disponíveis. As pessoas que não tinham tal disponibilidade foram encaminhadas para uma lista de espera e foram atendidas no ano subseqüente. Portanto, este estudo foi a primeira tentativa de aprimorar o estudo de Silva et al. (2000) e ocorreu no ano 2003.

No primeiro contato com as estagiárias responsáveis pela intervenção, os participantes foram informados sobre os objetivos do grupo e da pesquisa que seria realizada. Eles assinaram um Termo de Consentimento Pós-Informado, que estava de acordo com a Resolução do Conselho Nacional de Saúde no 196, de 10 de outubro de 1996. Esse projeto foi aprovado pelo Comitê de Ética da universidade em que foi desenvolvido.

O tratamento e a análise dos dados buscaram comparar as avaliações pré e pós-intervenção, conforme indicação de cada um dos instrumentos, tendo sido realizadas análises quantitativa e qualitativa. Com relação ao QHSE-P, foram atribuídos escores para as respostas dos participantes (a "freqüentemente", foi atribuído o escore 2, a "algumas vezes", o escore 1 , para as respostas "nunca ou quase nunca", o escore 0 , e, para as questões de especificidades do QHSE-P (p. ex. situações/assuntos), foram identificados os itens relatados pelos participantes e realizada análise de conteúdo. Para o IHS-Del Prette, os dados foram analisados conforme orientação do manual.

\section{Caracterização da intervenção}

As sessões de intervenção ocorreram no ano 2003 (vinculado a Estágio Supervisionado "Terapia Comportamental em Grupo I e II"), em dois grupos simultâneos, num total de 22 sessões de intervenções e 8 sessões de avaliação (quatro para medidas pré-teste e quatro para pós-teste).
A partir das avaliações iniciais, foram estabelecidos objetivos para cada indivíduo, os quais nortearam as intervenções. Cada sessão possuía esquematicamente a seqüência: a) a primeira parte, investigação das tarefas de casa e acontecimentos da semana, que busca descrever antecedentes, respostas e conseqüentes, identificar dificuldades e realizar análises funcionais (Sturmey, 1996); b) a segunda parte, que às vezes ocorria juntamente com a parte inicial, treino de habilidades sociais (por meio de diversas técnicas, tais como modelação, modelagem, reforçamento e role-playing); b) na seqüência, foram ampliadas habilidades, com base na literatura da área (Bolsoni-Silva, 2003; Del Prette \& Del Prette, 1999; Del Prette \& Del Prette, 2001b) e a partir dos diagnósticos realizados na fase de avaliação; c) passo seguinte, exposição teórica dialogada acerca do tema, momento em que novamente foram identificadas dificuldades, realizadas análises funcionais e treinados repertórios por meio de role-playings e/ou por vivências (Del Prette \& Del Prette, 2001b); d) as sessões eram finalizadas com a avaliação dos procedimentos e com a solicitação de tarefas para casa, que podiam ser gerais e/ou específicas para cada participante.

Destaca-se a relevância das avaliações diagnósticas, que permitiram conhecer previamente o repertório de habilidades sociais educativas dos participantes, colaborando na elaboração do programa no que diz respeito ao ajuste das necessidades de cada membro do grupo. Tal procedimento também favoreceu o conhecimento prévio dos recursos comportamentais dos participantes, que puderam ser utilizados durante as sessões na resolução de problemas relatados pelos membros dos grupos.

A primeira sessão foi destinada à discussão da habilidade social educativa de iniciar e manter conversações. Nas segunda, terceira e quarta sessões, foi discutida a expressão de 
sentimentos positivos. Na quarta sessão, foi iniciado o tema direitos humanos, que teve seqüência nas sessões 5 e 6 . As sessões 7 e 8 destinaram-se ao tema feedback positivo e negativo. A nona sessão, após o período de recesso escolar, retomou assuntos discutidos até aquele momento, que consistiu em oportunidade para os participantes relatarem dificuldades enfrentadas nesse período. Quatro sessões foram destinadas à diferenciação de comportamento não-habilidoso passivo, não-habilidoso ativo e habilidoso (sessões de 9 a 12). A sessão 14 destinou-se ao tema fazer perguntas, e, nas sessões 15 e 16, trabalhou-se a expressão de sentimentos negativos. As demais sessões (de 17 a 22) preocuparam-se em discutir estratégias socialmente habilidosas para estabelecer limites, incluindo expressar sentimento positivo, expressar sentimento negativo, expressar opinião, solicitar mudança de comportamento e apresentar consistência nas práticas educativas parentais.

Os participantes freqüentaram os grupos na seguinte proporção: a) 86,4\% para P8; b) P6 e P9 participaram de 81,8\% dos encontros; c) 77,3\% para P2 e P4; d) P7 freqüentou $72,8 \%$ e P3, 68,2\%; e) P1 e P5 freqüentaram 50\% das sessões.

\section{Resultados}

Nesta seção, encontram-se os resultados referentes às comparações das avaliações antes e após a intervenção, organizadas conforme os instrumentos utilizados: inventário de habilidades sociais (IHS-Del Prette) e questionário de habilidades sociais educativas parentais (QHSE-P).

O escore total das habilidades sociais de cada participante, mensurado pelo IHS-Del Prette, é apresentado na Figura 1.

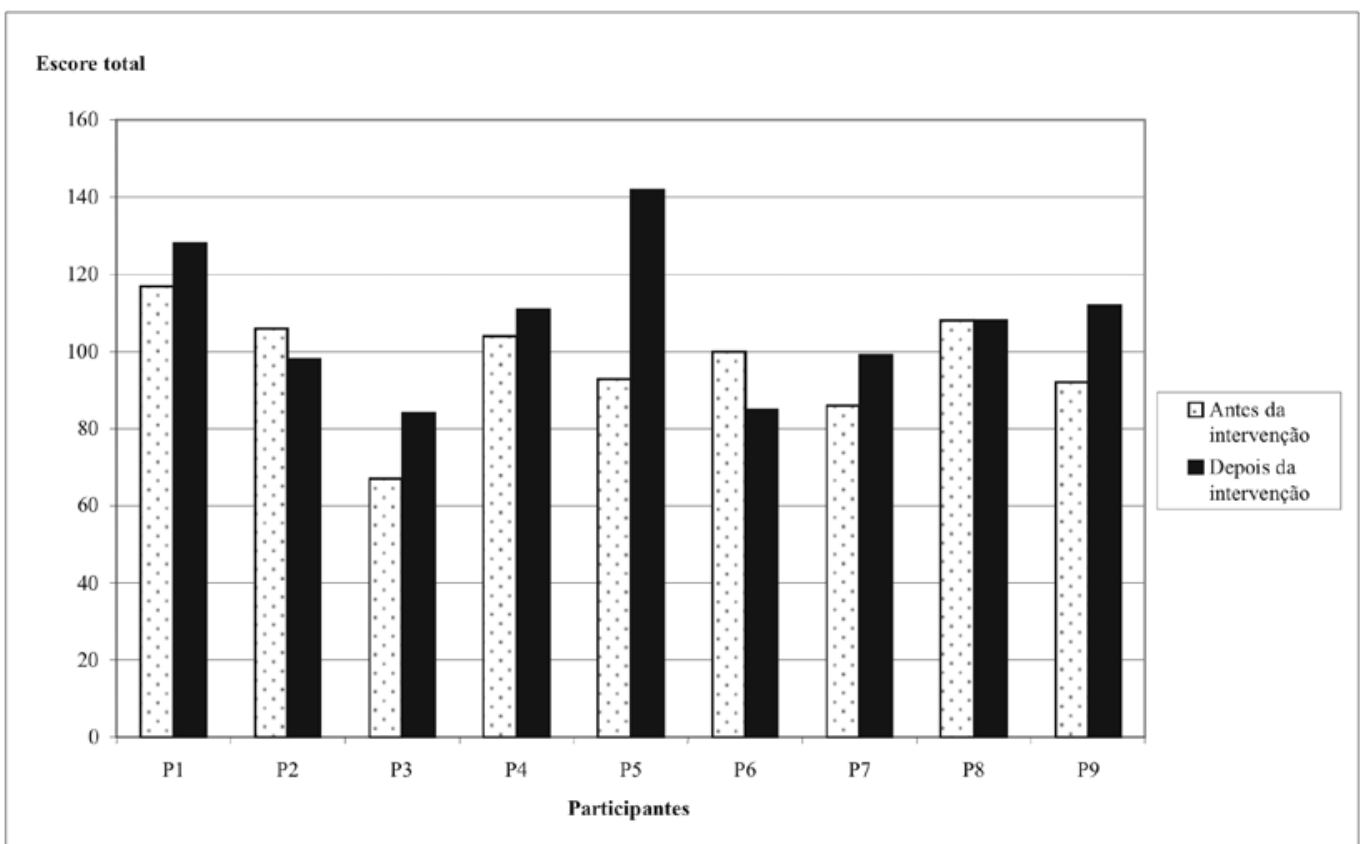

Figura 1. Escore geral do IHS, por participante, antes de depois da intervenção.

Pela Figura 1, observa-se um aumento no escore de seis participantes (P1, P3, P4, P5, P7 e P9), com destaque para a participante P5, cujo escore passou de 90 para 140 . O escore de 
P8 manteve-se inalterado nas medidas antes e após a intervenção, e houve uma redução nos escores de P6 e de P2.

$\mathrm{Na}$ análise por itens do IHS-Del Prette, destaca-se um aumento em 20 dos 34 itens avaliados $(58,8 \%$ \%). Cabe ressaltar que 12 itens já apresentavam um escore alto no pré-teste ( $\geq 3)$ : elogiar outrem, agradecer elogios, participar de conversação, falar a público desconhecido, discordar de grupo, devolver mercadoria defeituosa, recusar pedidos abusivos, elogiar familiares, fazer pergunta a conhecido, cumprimentar desconhecidos, pedir ajuda a amigos e negociar o uso de preservativo. Esses dados sugerem alto escore de habilidades sociais já no pré-teste e ainda assim, no pósteste, houve aumento e/ou manteve o escore $\geq 3$ em 11 desses 12 itens. Alguns itens, porém, diminuíram o escore no pós-teste: pedir mudança de comportamento, interromper a fala dos outros, elogiar outrem, participar de conversação, discordar de autoridade, declarar sentimento amoroso, devolver mercadoria defeituosa, recusar pedidos abusivos, fazer pergunta a conhecido, pedir ajuda a amigos, pedir favores a colegas e lidar com chacotas. Apenas o item discordar de grupo permaneceu com o mesmo escore no pré e no pós-teste.

A Figura 2 apresenta o escore médio para cada item avaliado no QHSE-P e compara as medidas pré e pós-intervenção para cada participante.

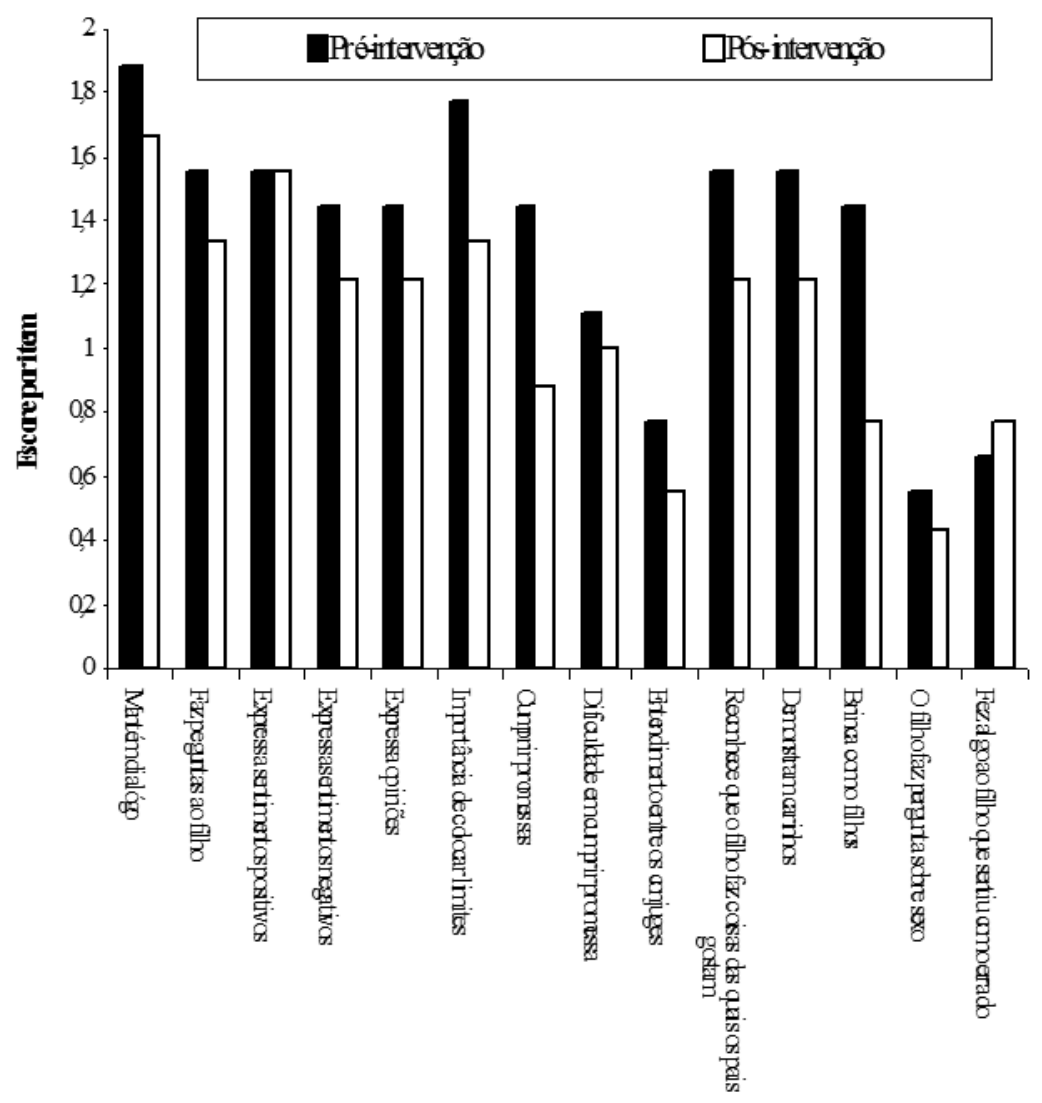

\section{Itens de Habilidades Sociais Educativas Parentais}

Figura 2. Escore médio por item de habilidades sociais educativas parentais 
A Figura 2 mostra que, dentre os 14 itens investigados, 10 foram avaliados com um escore alto no pré-teste $(\geq 1,4)$, sendo eles: manter diálogo, fazer perguntas, expressar sentimentos positivos, expressar sentimentos negativos, expressar opiniões, considerar importante estabelecer limites, cumprir promessas, identificar "bons" comportamentos, demonstrar carinho, brincar com os filhos. Destes, apenas manter diálogo e expressar sentimentos positivos se mantiveram com escore $\geq 1,4$ no pós-teste. Apenas o item faz algo ao filho que sentiu como errado foi avaliado mais positivamente no pós-teste, ainda que não tenha chegado ao escore médio.

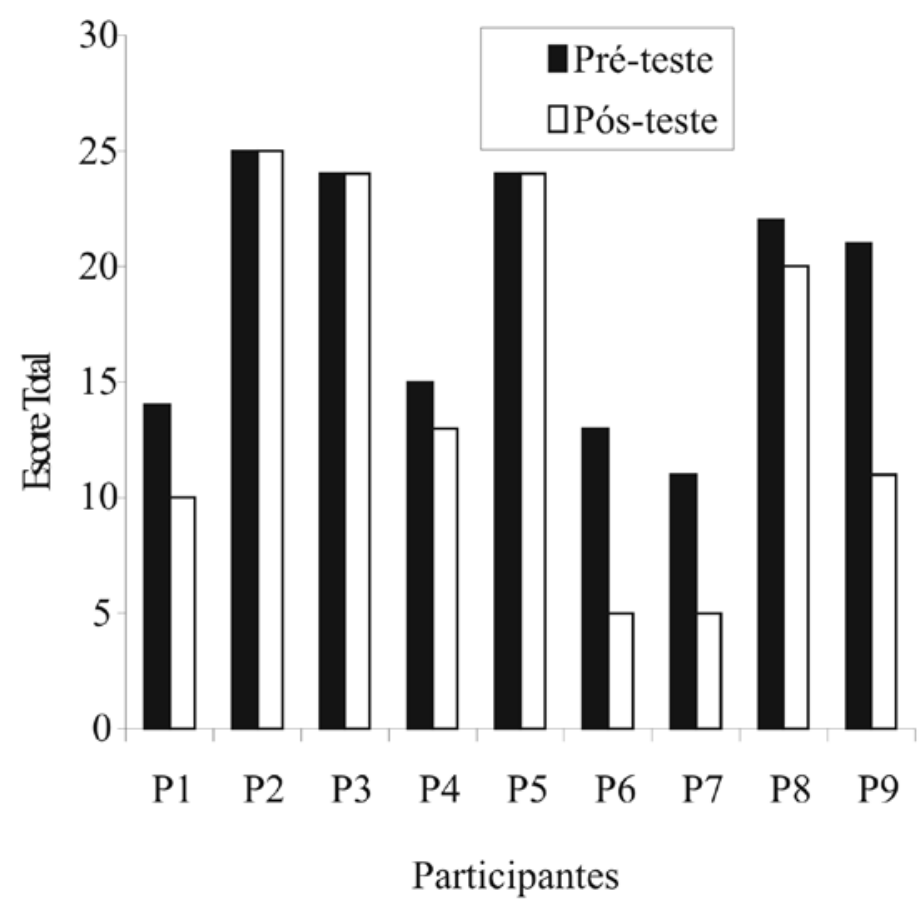

Figura 3. Escore total de cada participante

Na Figura 3, considerando que o escore máximo a ser atingido com o QHSE-P era o de valor 28 , verifica-se que quatro dos nove participantes apresentaram um escore alto $(\geq$ 20) antes da intervenção e o mantiveram após a mesma (P2, P3, P5, P8). Os participantes $\mathrm{P} 1, \mathrm{P} 4, \mathrm{P} 6$ e $\mathrm{P} 7$ relataram baixos escores nas medidas prévias à intervenção, que foram ainda mais reduzidos no pós-teste. Já P9 teve um escore alto no pré-teste ( $\geq 20)$, caindo para o escore 10 na segunda avaliação.

A partir das Figuras 1 e 2, conclui-se que: a) os participantes P3 e P5 aumentaram, no pós-teste, os escores no IHS-Del Prette e mantiveram-no no QHSE-P; b) P2, P4 e $\mathrm{P} 8$ praticamente mantiveram os escores em ambos os instrumentos; c) houve um aumento no IHS-Del Prette e uma redução no QHSE-P para P1, P7 e P9. É interessante destacar P6, que teve uma redução na pós-intervenção nos dois instrumentos.

Cabe ressaltar que alguns relatos dos participantes, durante as sessões, ilustraram aquisições de habilidades. Por exemplo, P3 relatou que, antes de freqüentar o grupo, 
tinha dificuldade em lidar com a ausência de comportamentos de carinho do filho, bem como com a alta freqüência de comportamentos considerados "indesejados", como não fazer as tarefas escolares.. No entanto, na avaliação após a intervenção, a cliente relatou estar mais sensível às "qualidades do filho", como saber dançar, e que, assim, ela pôde perceber que, ao diminuir suas cobranças excessivas e elogiar cada mudança positiva do filho (p. ex., melhora na escola), o mesmo estava "aproximando-se dela". A cliente descreveu que percebia a "aproximação" do filho quando o mesmo iniciava conversações quando ela estava cozinhando, momento em que também a convidava para cantar com ele.

Nota-se, ainda, na Tabela 2, que, na categoria "estratégias parentais para estabelecer limites", após a intervenção, essa cliente relatou que não mais colocava o filho de castigo e que procurava conversar e fazer pedidos. Os relatos dos participantes nas sessões também caracterizaram aquisições de habilidades mesmo para os que apresentaram o mesmo escore antes e depois; P2 relatou que, após as sessões, obteve o ganho de envolver o marido na educação na filha e ficou "tranqüila" por ter o hábito de evitar práticas punitivas ao educar, uma vez que era comumente questionada por seu marido e por sua mãe quanto às suas práticas "não punitivas".

A análise das especificidades de HSE-P, apresentadas na Tabela 2, apontou algumas diferenças no pós-teste quando comparadas às das medidas pré-teste. Outras que foram freqüentemente apontadas já no pré-teste mantiveram a mesma freqüência na segunda avaliação: manter diálogo, fazer perguntas, expressar sentimentos negativos, entendimento conjugal, demonstrar carinho, brincar com o filho, ouvir perguntas sobre sexualidade e admitir erro.

Os relatos dos participantes nas sessões também caracterizaram aquisições de habilidades mesmo para os que apresentaram o mesmo escore antes e depois; P2 relatou que, após as sessões, obteve o ganho de envolver o marido na educação na filha e ficou "tranqüila" por ter o hábito de evitar práticas punitivas ao educar, uma vez que era comumente questionada por seu marido e por sua mãe quanto às suas práticas "não punitivas". 
Tabela 2. Caracterização dos temas trabalhados em cada sessão, bem como os participantes presentes

\begin{tabular}{|c|c|c|}
\hline Sessão & Tema trabalhado & Participantes presentes \\
\hline \multirow[t]{3}{*}{$1 \underline{\underline{a}}$} & Iniciar e manter conversações. & $\mathrm{P} 1, \mathrm{P} 2, \mathrm{P} 3, \mathrm{P} 5$ \\
\hline & Observar comportamentos dos pais e seus & \\
\hline & efeitos na interação com os filhos & P6, P7, P8 e P9 \\
\hline \multirow[t]{2}{*}{$2^{\mathrm{a}}$} & Expressar sentimento positivo & $\mathrm{P} 1, \mathrm{P} 2, \mathrm{P} 4$ \\
\hline & Observar e dar feedback positivo & P6, P7, P8 e P9 \\
\hline $3^{\underline{a}}$ & Expressar sentimento positivo & $\mathrm{P} 1, \mathrm{P} 4, \mathrm{P} 6, \mathrm{P} 7$ e $\mathrm{P} 8$ \\
\hline \multirow[t]{2}{*}{$4 \underline{a}$} & Expressar sentimento positivo & P1, P2, P3,P4, P5 \\
\hline & Discriminar acerca de direitos humanos & P6, P7, P8 e P9 \\
\hline $5^{\mathrm{a}}$ & Discriminar acerca de direitos humanos & $\mathrm{P} 1, \mathrm{P} 2, \mathrm{P} 3, \mathrm{P} 4, \mathrm{P} 5, \mathrm{P} 8$ e P9 \\
\hline $6^{\underline{a}}$ & Discriminar acerca de direitos humanos & $\mathrm{P} 1, \mathrm{P} 3, \mathrm{P} 4, \mathrm{P} 5 \mathrm{P} 8$ e P9 \\
\hline $7 \underline{a}$ & Dar feedback positivo e negativo & $\mathrm{P} 2, \mathrm{P} 4, \mathrm{P} 6, \mathrm{P} 8$ e P9 \\
\hline $8^{\mathrm{a}}$ & Dar feedback positivo e negativo & $\mathrm{P} 1, \mathrm{P} 2, \mathrm{P} 3, \mathrm{P} 5, \mathrm{P} 6, \mathrm{P} 7$ e P8 \\
\hline 9a & $\begin{array}{l}\text { Avaliar os encontros anteriores (retorno das férias) } \\
\text { e relatar as dificuldades }\end{array}$ & P1, P2, P3, P6, P7, P8 e P9 \\
\hline $10^{\mathrm{a}}$ & $\begin{array}{l}\text { Discriminar efeitos de práticas educativas punitivas } \\
\text { Discriminar acerca de comportamentos passivo, } \\
\text { agressivo e assertivo/habilidoso }\end{array}$ & P2, P3, P4, P5, P6, P7, P8 e P9 \\
\hline $11 \underline{a}$ & $\begin{array}{l}\text { Discriminar efeitos de práticas educativas punitivas } \\
\text { Discriminar acerca de comportamentos passivo, } \\
\text { agressivo e assertivo/habilidoso }\end{array}$ & P2, P3, P4, P6, P7 e P9 \\
\hline $12^{\mathrm{a}}$ & $\begin{array}{l}\text { Discriminar acerca de comportamentos passivo, } \\
\text { agressivo e assertivo/habilidoso }\end{array}$ & $\mathrm{P} 1, \mathrm{P} 2, \mathrm{P} 3, \mathrm{P} 4, \mathrm{P} 5, \mathrm{P} 6, \mathrm{P} 7, \mathrm{P} 8$ e P9 \\
\hline $13^{\mathrm{a}}$ & $\begin{array}{l}\text { Discriminar acerca de comportamentos passivo, } \\
\text { agressivo e assertivo/habilidoso }\end{array}$ & $\mathrm{P} 2, \mathrm{P} 4, \mathrm{P} 6, \mathrm{P} 7$ e P9 \\
\hline $14^{\mathrm{a}}$ & $\begin{array}{l}\text { Discriminar próprios comportamentos e seus efeitos } \\
\text { para a interação com os filhos Fazer perguntas, } \\
\text { pedir esclarecimento }\end{array}$ & $\mathrm{P} 2, \mathrm{P} 4, \mathrm{P} 6$ e P9 \\
\hline $15^{\underline{a}}$ & $\begin{array}{l}\text { Estabelecer limites } \\
\text { Expressar sentimento negativo }\end{array}$ & $\begin{array}{l}\text { P1, P2, P3, P4, } \\
\text { P6, P7, P8 e P9 }\end{array}$ \\
\hline $16^{\mathrm{a}}$ & $\begin{array}{l}\text { Estabelecer limite } \\
\text { Expressar sentimento negativo }\end{array}$ & $\begin{array}{l}\text { P2, P3, P4, P5, } \\
\text { P6, P7 e P8 }\end{array}$ \\
\hline $17^{a}$ & Estabelecer limite & $\mathrm{P} 2, \mathrm{P} 3, \mathrm{P} 4, \mathrm{P} 5, \mathrm{P} 8$ e P9 \\
\hline $18^{\underline{a}}$ & Estabelecer limite & $\mathrm{P} 1, \mathrm{P} 2, \mathrm{P} 3, \mathrm{P} 4, \mathrm{P} 6, \mathrm{P} 7, \mathrm{P} 8$ e P9 \\
\hline $19^{a}$ & $\begin{array}{l}\text { Discriminar estratégias para haver consistência conjugal } \\
\text { Estabelecer limite e relacionar-se com autoridade }\end{array}$ & $\begin{array}{l}\text { P2, P3, P4, P5 } \\
\text { P6, P7, P8 e P9 }\end{array}$ \\
\hline $20^{\mathrm{a}}$ & $\begin{array}{l}\text { Discriminar estratégias para haver consistência conjugal } \\
\text { Estabelecer limite e relacionar-se com autoridade }\end{array}$ & $\begin{array}{l}\text { P3, P4, P5 } \\
\text { P6, P7, P8 e P9 }\end{array}$ \\
\hline $21^{\mathrm{a}}$ & $\begin{array}{l}\text { Estabelecer limite e relacionar-se com autoridade } \\
\text { Expressar sentimento positivo }\end{array}$ & $\mathrm{P6}, \mathrm{P7}, \mathrm{P} 8$ e $\mathrm{P9}$ \\
\hline $22^{\mathrm{a}}$ & $\begin{array}{l}\text { Estabelecer limite e relacionar-se com autoridade } \\
\text { Expressar sentimento positivo }\end{array}$ & P8 e P9 \\
\hline
\end{tabular}


Na categoria expressar sentimento positivo, dos nove participantes, cinco indicaram um maior número de especificidades (P2, P4, P5, P6, P7), três clientes mantiveram o mesmo número de comportamentos descritos (P1, P3 e P9) e apenas P8 citou cinco comportamentos na avaliação pré-intervenção e quatro na avaliação pós-teste. Em expressar opinião, destaca-se a redução na freqüência de relatos quanto a concepções de certo e errado e aumento de outras categorias (falar sobre amizades e religião). Na categoria estratégias parentais para estabelecer limites, verifica-se que todos os participantes descreveram um menor número de práticas punitivas após a intervenção terapêutica. Cabe ressaltar que, enquanto na fase de pré-intervenção poucos clientes (P1, P8, P9) relataram conversar para estabelecer limites, na segunda avaliação, sete relataram utilizar tal estratégia. De modo semelhante, fazer pedidos foi apontado primeiramente por poucos participantes, aumentando para sete a freqüência no pósteste. A descrição de sentimentos correlatos ao estabelecer limites também sinaliza uma melhora na interação, pois, consensualmente, todos os participantes relataram sentirem-se "bem" no pós-teste, o que não foi identificado na primeira avaliação. Quanto a dificuldades em cumprir promessas, observou-se uma redução considerável no pós-teste, o que sugere aquisição de repertório. Nos itens relacionados às habilidades e qualidades do filho, também todos os participantes indicaram um maior número de itens após a intervenção terapêutica, exceto as clientes $\mathrm{P} 4$ e P5. A participante P9 manteve as mesmas descrições.

Outras que foram freqüentemente apontadas já no pré-teste mantiveram a mesma freqüência na segunda avaliação: manter diálogo, fazer perguntas, expressar sentimentos negativos, entendimento conjugal, demonstrar carinho, brincar com o filho, ouvir perguntas sobre sexualidade e admitir erro.

\section{Discussão}

De maneira geral, os resultados indicam a aquisição de algumas habilidades sociais educativas promovidas diretamente pelo programa: agradecer a elogio, reagir a elogio, expressar sentimento positivo, elogiar familiares, expressar sentimento positivo, recusar pedido abusivo, manter conversação, evitar o uso de práticas punitivas e estabelecer limites de forma mais positiva. Algumas habilidades identificadas no IHS-Del Prette são sinalizadoras de generalização: manter conversação com desconhecido (com e sem interesse de envolvimento amoroso), falar a público conhecido e fazer pergunta a desconhecido, encerrar conversação, abordar autoridade, encerrar conversa ao telefone, lidar com críticas dos pais, fazer pergunta a desconhecido e pedir favores a desconhecidos, expressar desagrado a amigos e negociar o uso de preservativo. A aquisição dessas habilidades sociais indica uma melhoria na quantidade e na qualidade de interações estabelecidas entre pais e filhos e também com outras pessoas de sua convivência, o que sugere um efeito positivo do programa de intervenção descrito e avaliado.

Chama a atenção o fato de que, para algumas habilidades e para alguns participantes, escores no pós-teste apresentaram redução em comparação com o pré-teste, como por exemplo, em relação aos participantes $\mathrm{P} 2$ e P6, cujo escore geral do IHS-Del Prette ficou reduzido após a intervenção. Pode-se pensar em algumas possíveis explicações, e a primeira sugere que, como os instrumentos são de autorelato, os participantes, ao se avaliarem, podem ter superestimado seus desempenhos na primeira avaliação, ou seja, a intervenção pode ter propiciado a esses indivíduos uma situação de autoconhecimento e de discriminação de seus próprios comportamentos diante das demandas. Conforme Skinner (1953/1993), o autoconhecimento é necessário para que a pessoa seja capaz de automonitorar-se e de autocontrolar-se, e apresenta comportamentos precorrentes para a emissão de respostas que aumentem a probabilidade de obter reforçadores em suas interações sociais.

A intervenção ora mencionada primou por ajudar os participantes a descreverem 
funcionalmente seus comportamentos e os de seus filhos, o que pode ter contribuído para uma avaliação mais rigorosa no pósteste. Por outro lado, cabe lembrar que os participantes não possuíam queixas clínicas e que procuraram o grupo a fim de conhecer mais sobre o relacionamento estabelecido entre pais e filhos. Não é surpreendente, portanto, que, já na primeira avaliação, apresentassem altos escores de habilidades sociais educativas. Entretanto, é interessante notar que, ainda assim, essas pessoas puderam ser beneficiadas por um programa de intervenção quanto à aquisição de habilidades e melhoria do relacionamento com os filhos. Bolsoni-Silva (2003) verificou que pais e mães de crianças sem queixas clínicas relatavam emitir, com mais freqüência, habilidades sociais educativas; assim, é possível que as pessoas que procuraram por esse atendimento já tivessem esse perfil.

Cabe ressaltar a presença dos pais do sexo masculino nesse grupo. Apesar do número reduzido (apenas dois), os mesmos mostraram interesse em buscar conhecimento para uma tarefa que é usualmente atribuída às mulheres. Os estudos de Silva et al. (2000) e de BolsoniSilva (2003) indicam que, do ponto de vista de pais e de mães, são estas que participam mais da educação dos filhos. Bolsoni-Silva (2003) encontrou diferenças na forma como pais e mães se relacionam com os filhos, sendo estatisticamente mais freqüentes às mães habilidades sociais educativas de comunicação (iniciar e manter conversação, fazer perguntas) e de expressividade (de sentimentos positivos e negativos e de opiniões); os pais relataram, mais freqüentemente que as mães, brincar com os filhos, especialmente nos finais de semana e cumprir promessas. Esses resultados sugerem a relevância de futuros estudos que possam comparar intervenções realizadas apenas com mães ou com pais ou mistas, de forma a atender diferencialmente e garantir a promoção de todas as habilidades sociais educativas parentais necessárias para ambos os progenitores.

A população mencionada, como visto na Tabela 2, utilizava freqüentemente estratégias coercitivas para estabelecer limites. Esse resultado vai ao encontro dos dados obtidos por Bolsoni-Silva (2003), que verificou que familiares de crianças com e sem problemas de comportamento utilizavam de modo semelhante tal estratégia. No entanto, as medidas de pós-teste do questionário de habilidades sociais educativas parentais apontou uma redução de comportamentos coercitivos e o aumento de outras habilidades, como conversar e fazer pedidos. As habilidades sociais educativas relacionadas à expressão de sentimento positivo, efeitos de práticas punitivas e estabelecimento de limites foram amplamente treinadas nessa intervenção, o que justifica os resultados encontrados nessa direção.

Sidman (1995) e Skinner (1953/1993) acreditavam que o uso de práticas agressivas (p. ex. bater, gritar) gera ansiedade e baixa auto-estima, além de ensinar aos filhos modelos agressivos de lidar com conflitos e a tendência de contra-controlar (p. ex. com desobediência e agressividade). Concordando com esses pesquisadores, o uso da agressividade pode e deve ser evitado na educação com os filhos, pois é possível estabelecer limites valendose de estratégias claramente mais positivas, identificadas em estudos prévios, como preventivas de problemas de comportamento e que precisam ser informadas e treinadas amplamente com pais e com mães, de forma a possibilitar a mudança dessa prática cultural historicamente incentivada. Na mesma direção, Pacheco, Teixeira e Gomes (1999) apontam a necessidade de os pais e as mães serem responsivos a seus filhos, isto é, garantirem também o afeto, a atenção e o carinho bem como procurarem formas não agressivas de estabelecer limites.

A intervenção avaliada neste trabalho apresenta algumas aproximações com a literatura da área: a) foi realizada em grupo e com avaliações pré e pós-teste (p. ex., Dishion \& Andrews, 1995; Pfiffner \& McBurnett, 1997; Ruma et al., 1996); b) ocorreu uma vez por semana (p. ex., Brestan et al., 1999; Cobham et al., 1998; Peterson et al., 2003; Sanders et al., 2000; 
Taylor et al., 1998); c) treinou habilidades de manejo de comportamento e de promoção de competência social (p. ex., Brestan et al., 1999; Dishion \& Andrews, 1995; Jouriles et al., 2001; Ruma et al., 1996; Sanders et al., 2000).

Cabe ressaltar que o programa de intervenção utilizado, apesar de considerar o manejo do comportamento da criança durante todo o processo, teve como foco a promoção de habilidades sociais educativas dos pais.

Comparando esta pesquisa com a anterior (Silva et al. 2000), verifica-se que esta intervenção supriu algumas lacunas no conhecimento. Silva et al. (2000) relataram um estudo que foi realizado mediante poucas sessões (num total de 10) que eram altamente estruturadas, isto é, com pouca flexibilidade frente às demandas diárias dos participantes. Além disso, a avaliação, apesar de investigar diversas habilidades sociais, deixou de investigar funcionalmente os repertórios sociais, o que dificulta um diagnóstico comportamental mais completo.
Ainda que com resultados promissores, deve-se evitar generalizar os resultados para outros grupos, uma vez que este programa foi conduzido com um número reduzido de participantes. Estudos futuros poderiam preocupar-se em avaliar também os comportamentos dos filhos, cuja medida daria pistas indiretas de generalização das habilidades dos pais bem como de seus efeitos.

Avaliações de seguimento deveriam ser realizadas, com seis meses e/ou um ano, em que os mesmos instrumentos de coleta fossem reaplicados com o intuito de monitorar as aquisições de repertórios identificados no pós-teste e verificar a permanência ou não dos ganhos obtidos. Poucos estudos foram conduzidos com esse delineamento (Pfiffner \& McBurnett, 1997; Sanders et al., 2000).

Outra sugestão para futuras pesquisas refere-se à realização de comparações entre grupos experimentais e grupos-controle, de forma a mensurar a variável interveniente tempo; também são poucos os trabalhos que mencionam tal delineamento (Dishion e Andrews, 1995; Jouriles et al., 2001; Peterson et al., 2003; Sanders et al., 2000).

Alessandra Turini Bolsoni-Silva

Doutora em Psicologia e docente do Departamento de Psicologia da Universidade Estadual Paulista (UNESP-Bauru).

E-mail: bolsoni@yahoo.com.br

\section{Alessandra Salina-Brandão}

Psicóloga, mestre em Educação Especial e docente substituta do Departamento de Psicologia da Universidade Estadual Paulista.

E-mail: alessandrasalina@hotmail.com

\section{Andréa Regina Rosin-Pinola}

Psicóloga e doutoranda em Psicologia da Universidade de São Paulo (USP-Ribeirão Preto).

E-mail: rosin andrea@hotmail.com

\section{Fabiana Maris Versuti-Stoque}

Psicóloga e mestranda em Educação para a ciência da Universidade Estadual Paulista (UNESP-Bauru).

E-mail: faversuti@hotmail.com

Endereço para correspondência:

Rua Paraná, no 3-39, Jardim Coralina Cep 17030-023, Bauru-SP 


\section{Referências}

Bolsoni-Silva, A. T. (2003). Habilidades sociais educativas, variáveis contextuais e problemas de comportamento: comparando pais e mães de pré-escolares. Tese de Doutorado. Curso de Pós-Graduação em Ciências, Universidade de São Paulo, Ribeirão Preto- SP.

Bolsoni-Silva, A. T., \& Del Prette, A. (2002). O que os pais falam sobre suas habilidades sociais e de seus filhos? Argumento, 4(7), 71-86

Bolsoni-Silva, A. T., \& Marturano, E. M. (2006). A qualidade da interação entre pais e filhos e sua relação com problemas de comportamento de pré-escolares In Estudos sobre habilidades sociais e relacionamento interpessoal (pp. 89-104). São Paulo: Casa do Psicólogo.

Bolsoni-Silva, A. T., \& Marturano, E. M. (2002). Práticas educativas e problemas de comportamento: uma análise à luz das habilidades sociais. Estudos de Psicologia, 7(2), 227-235.

Bolsoni-Silva, A. T., Del Prette, A., \& Oishi, J. (2003). Habilidades sociais de pais e problemas de comportamento de filhos. Revista Psicologia Argumento, 9, 11-29.

Brestan, E. V., Jacobs, J. R., Rayfield, A. D., \& Eyberg, S. M. (1999). A consumer satisfaction measure for parent-child treatments and its relation to measures of child behavior change. Behavior Therapy, 30, 17-30.

Cobham, V. E., Dadds, M. R., \& Spence, S. H. (1998). The role of parental anxiety in the treatment of childhood anxiety. Journal of Consulting \& Clinical Psychology, 66(6), 893-905.

Del Prette, Z. A. P., \& Del Prette, A. (1999). Psicologia das habilidades sociais: terapia e educação. Petrópolis RJ, :Vozes.

Del Prette, Z. A. P., \& Del Prette, A. (2001a). Inventário de habilidades sociais (IHS-Del Prette-Del-Prette): manual de aplicação, apuração e interpretação. São Paulo: Casa do Psicólogo.

Del Prette, Z. A. P., \& Del Prette, A. (2001b). Psicologia das relações interpessoais. Vivências para o trabalho em grupo. Petrópolis: Vozes.

Dishion, T. J., \& Andrews, D. (1995). Preventing escalation in problem behaviors with high-risk young adolescents: immediate 1-year outcomes. Journal of Consulting \& Clinical Psychology, 63 (4), 538-548.

Gomide. P. I. C. (2006). Inventário de estilos parentais. modelo teórico: manual de aplicação,apuração e interpretação. Petrópolis RJ, :Vozes

Jouriles, E. N., McDonald, R., Spiller, L., Norwood, W. D, Swank, P. R., \& Stephens, N. (2001). Reducing conduct problems among children of battered women. Journal of Consulting and Clinical Psychology, 69 (5), 774-785.

Kaplan, H. I., Sadock, B. J., \& Grebb, J. A. (1997). Compêndio de psiquiatria: ciências do comportamento e psiquiatria clínica. Porto Alegre: Artes Médicas.

Marinho, M. L. (1999). Comportamento infantil anti-social: Programa de intervenção junto à família. In R.R. Kerbauy, \& R. C Wielenska, . (Eds.). Sobre comportamento e cognição: Psicologia comportamental e cognitiva - da reflexão teórica à diversidade na aplicação. (Vol. 4 pp. 207-215). São Paulo: Arbytes.

McMahon, R. J. (1996). Treinamento de pais. In V. E. Caballo, (Ed.). Manual de técnicas de terapia e modificação do comportamento. (pp. 397-424). São Paulo: Livraria Editora Santos.

Pacheco, J. T. B., Teixeira, M. A. P., \& Gomes, W. B. (1999). Estilos parentais e desenvolvimento de habilidades sociais na adolescência. Psicologia: Teoria e Pesquisa, 15(2), 117-126

Peterson, L., Tremblay, G., Ewigman, B., \& Saldana, L. (2003). Multilevel selected primary prevention of child maltreatment. Journal of Consulting and Clinical Psychology, 71(3), 601-612.

Pfiffner, L., \& McBurnett, K. (1997). Social skills training with parent generalization: treatment effects for children with attention deficit disorder. Journal of Consulting and Clinical Psychology, 65 (5), 749-757.

Rocha, M. M., \& Brandão, M. Z. da S. A importância do autoconhecimento dos pais na análise e modificação de suas interações com os filhos. In M. Delitti (Ed.), Sobre comportamento e cognição. (Vol. 2, pp. 137-146). São Paulo: ARBytes Editora.

Ruma, P. R., Burke, R. V., \& Thompson, R. W. (1996). Group parent training: Is it effective for children of all ages? Behavior Therapy, 27, 159-169.

Sanders, M. R., Markie-Dadds, C., Tully, L. A. \& Bor, W. (2000). The triple p-positive parenting program: a comparison of enhanced, standard, and self-directed behavioral family intervention for parents of children with early onset conduct problems. Journal of Consulting and Clinical Psychology, 68 (4), 624-640.

Sidman, M. (1995). Coerção e suas implicações. Campinas, SP: Editorial Psy II.

Silva, A. T. B. (2000). Problemas de comportamento e comportamentos socialmente adequados: sua relação com as habilidades sociais educativas de pais. Dissertação de Mestrado. Curso de Pós-Graduação em Educação Especial. Universidade Federal de São Carlos. São Carlos-SP.

Silva, A. T. B., Del Prette, A., \& Del Prette, Z. A. P. (2000). Relacionamento pais-filhos: um programa de desenvolvimento interpessoal em grupo. Psicologia Escolar e Educacional, 3(3), 203-215.

Skinner, B. F. (1993). Ciência e comportamento humano. São Paulo: Martins Fontes. (Trabalho original publicado em 1953)

Sturmey, P. (996). Functional analysis in clinical Psychology. England: John Wiley \& Sons, 1996.

Taylor, T. K., Schmidt, F., Pepler, D., \& Hodgins, C. (1998). A comparison of ecletic treatment with Webster-Stratton's parents and children series in a chidren's mental health center: A randomized controlled trial. Behavior Therapy, 29, 221-240.

Webster-Stratton, C. (1994). Advancing videotape parent training: A comparison study. Journal of Consulting and Clinical Psychology, 62(3), 583-593. 